Collagen degradation and preservation of MMP-8 activity in human dentine matrix after demineralization

\title{
Hedenbjork-Lager, Anders
}

2016-08

Hedenbjork-Lager , A , Hamberg , K , Paakkonen , V , Tjaderhane , L \& Ericson , D 2016 , ' Collagen degradation and preservation of MMP-8 activity in human dentine matrix after demineralization ' , Archives of Oral Biology , vol. 68 , pp. 66-72 . https://doi.org/10.1016/j.archoralbio.2016.04.003

http://hdl.handle.net/10138/224109

https://doi.org/10.1016/j.archoralbio.2016.04.003

publishedVersion

Downloaded from Helda, University of Helsinki institutional repository.

This is an electronic reprint of the original article.

This reprint may differ from the original in pagination and typographic detail.

Please cite the original version. 


\title{
Collagen degradation and preservation of MMP-8 activity in human dentine matrix after demineralization
}

\author{
Anders Hedenbjörk-Lager ${ }^{a, *}, K^{2}$ ristina Hamberg ${ }^{a}$, Virve Pääkkönen ${ }^{b}$, Leo Tjäderhane ${ }^{\text {b,c,d }}$, \\ Dan Ericson $^{\mathrm{a}}$ \\ a Department of Cariology, Faculty of Odontology, Malmö University, Sweden \\ ${ }^{\mathrm{b}}$ Research Unit of Oral Health Sciences, University of Oulu, Oulu, Finland \\ ${ }^{\mathrm{c}}$ Medical Research Center, Oulu University Hospital and University of Oulu, Finland \\ d Department of Oral and Maxillofacial Diseases, University of Helsinki and Helsinki University Hospital, Helsinki, Finland
}

\section{A R T I C L E I N F O}

\section{Article history:}

Received 22 June 2015

Received in revised form 21 March 2016

Accepted 10 April 2016

\section{Keywords:}

Matrix metalloproteinase

Tooth demineralization

Edetic acid

Acetic acid

Collagen

\begin{abstract}
A B S T R A C T
Objective: Dental caries is a process driven by acids produced by oral microorganisms followed by degradation of the dentine collagen matrix by proteolytic enzymes. Matrix metalloproteinases (MMPs) have been suggested to contribute to caries by degrading collagen. The aim of this study was to develop a method for generating demineralized dentine matrix substrate (DDM) maintaining MMP-8 bioactivity and no interference with later assays. Such a substrate would allow study of the effects of various treatments on MMP-8 activity and collagen degradation in demineralized dentine.

Design: Human dentine was powderized in a tissue grinder and frozen $\left(-80^{\circ} \mathrm{C}\right)$. The powder was demineralized in dialysis tubes, using EDTA or acetic acid. The demineralized dentine matrix (DDM) was harvested and analyzed for collagen content using SDS-PAGE. The DDM was subsequently suspended in PBS or TESCA buffer. Protein, MMP-8 (ELISA) and collagen (HYP) was analyzed directly or after 1 wk. Results: EDTA or acid demineralization of dentine using dialysis yielded a substrate rich in collagen coupled with preserved MMP-8 activity. Collagen degraded in room temperature, assessed by higher HYP amounts in the soluble fraction of DDM after one wk, indicating that the methods used preserved active DDM-components after the demineralization process.

Conclusions: The presented demineralization methods both provided insoluble DDM substrates suitable for further intervention studies. However, it was found that the substrates differed depending on the demineralization method and buffers used. This needs further study to find an optimal technique for generating DDM with retained proteins as well as enzymatic bioactivity.
\end{abstract}

(c) 2016 Elsevier Ltd. All rights reserved.

\section{Introduction}

Dental caries is a process driven by acids produced by oral microorganisms as a consequence of their metabolism of fermentable carbohydrates (Takahashi \& Nyvad, 2011). The caries process in dentine can be divided into two distinct steps: (i) acid dissolution of the mineralized phase of the tooth and (ii) degradation of the dentine collagen matrix by proteolytic enzyme action.

Both steps have been attributed to the oral microflora despite the fact that the mechanisms behind dentine collagen degradation have been obscured. Degradation was for long thought to be caused

\footnotetext{
* Corresponding author at: Department of Cariology, Faculty of Odontology, Malmö University SE 20506 Malmö, Sweden.

E-mail address: anders.hedenbjork.lager@mah.se (A. Hedenbjörk-Lager).
}

by the action of bacterial acids or unspecific bacterial proteases. However, bacterial acids are unable to degrade intact collagen (Katz, Park, \& Palenik, 1987), and the oral microbiome lacks the enzymatic capacity to degrade intact collagen in vitro (van Strijp, van Steenbergen, de Graaff \& ten Cate, 1994; van Strijp, van Steenbergen \& ten Cate, 1997).

Matrix metalloproteinases (MMPs) are a group of endopeptidase enzymes produced by connective tissue cells (fibro-, osteoand odontoblasts) as well as by polymorphonuclear leukocytes and other inflammatory cells (Chaussain-Miller, Fioretti, Goldberg, \& Menashi, 2006). As a group they have the ability to degrade most extracellular matrix molecules (Visse \& Nagase, 2003).

Among the various species of MMPs that have been detected in dentine, MMP-8 is considered the major dentinal collagenolytic MMP (Sorsa, Tjäderhane \& Salo, 2004; Sulkala et al., 2007). The mechanism by which MMP-8 contributes to collagen degradation has been suggested to be a stepwise process: (i) - Hydroxyapatite 
is dissolved by bacterial acidic action, thereby revealing the previously mineral-protected collagen molecules as well as other extracellular matrix (ECM) components, such as proMMPs. (ii) The acid induced salivary $\mathrm{pH}$ drop to around 4 or 5 activates proMMP-8 (Tjäderhane et al., 1998). MMPs are neutral proteases, and not active at such a low $\mathrm{pH}$, but they can withstand even prolonged exposure to very acidic conditions without losing their bioactive properties (Tezvergil-Mutluay et al., 2013). (iii) - When $\mathrm{pH}$ is increased again due to the cyclical $\mathrm{pH}$ fluctuation in caries, the activated MMP-8 might degrade collagen. (iv) - The fragmented collagen - gelatin - can subsequently be degraded further by gelatinases (MMP-2 and-9) or non-specific proteases of bacterial or endogenous origin. It should be noted that most of the knowledge on dentine MMP-8 is derived from studies on dentine bonding degeneration.

There is a growing body of data supporting that MMPs play a role in dentine degradation. A recent cross-sectional clinical study demonstrated a very strong relationship between elevated salivary MMP-8 levels and dentine caries. Subjects with manifest cavities exhibited much higher salivary MMP-8 concentrations than subjects without caries (Hedenbjörk-Lager et al., 2015). The source of the salivary MMP- 8 could not be determined, but a plausible explanation could be that the MMP- 8 originated from carious dentine. Other studies indicating the importance of MMPs in dentine caries have demonstrated that specific inhibition of MMP activity can reduce the caries progression in rats (Sulkala et al., 2001; Tjäderhane et al., 1999). In vitro addition of active MMP-8 to saliva also influences remineralization negatively (Nordbø, Leirskar, Ngo, Mount, \& Wahlgren, 2003). To our knowledge, clinical studies on the role of endogenous proteolytic enzymes in the dentine caries process are lacking.

A complication with previous in vitro studies is the multitude of methods used for obtaining the demineralized dentine substrate and also the use of different demineralization procedures, thus making comparison of results difficult. To further study the mechanisms of endogenous dentine degradation a robust method for generation of dentin substrate that does not hamper or destroy the bioactivity of the target proteolytic enzymes is needed. In addition to being in a soluble phase, dentine powder has the advantage of a considerable surface area, thus permitting faster reactions as compared to similarly intended analyses of dentine beams (Carrilho et al., 2009).

In this study we wanted to be able to monitor the levels of MMP-8 after various treatments, as well as the levels of collagen degradation (via assessment of hydroxyproline [HYP] levels). Thus, the aims for this study were (i) to develop a method for generating a demineralized dentine matrix substrate from human dentine powder. (ii) To ensure that this substrate retain the major part of the insoluble collagen along with its associated proteins as well as the MMP-8 enzyme in active form.

The dentine substrate would then allow subsequent study of the effects of various treatments on MMP activity and collagen degradation in demineralized dentine substrate from caries-free teeth.

\section{Materials and methods}

\subsection{Dentine collection}

The teeth used for the dentine powder were erupted healthy wisdom teeth from patients aged 18-30 years, collected from the Oral Surgery unit in Oulu University, Finland. The teeth were extracted as part of the normal treatment, used with the patient's consent and approval from the Ethical Committee, Faculty of Medicine, University of Oulu protocol \#19/2006. After extraction the teeth were immediately collected and taken to the laboratory for processing. The enamel was removed using high-speed burs, under irrigation of copious amounts of water. The roots were separated from the crown and the dental pulp was removed, followed by meticulous scraping to remove the odontoblasts.

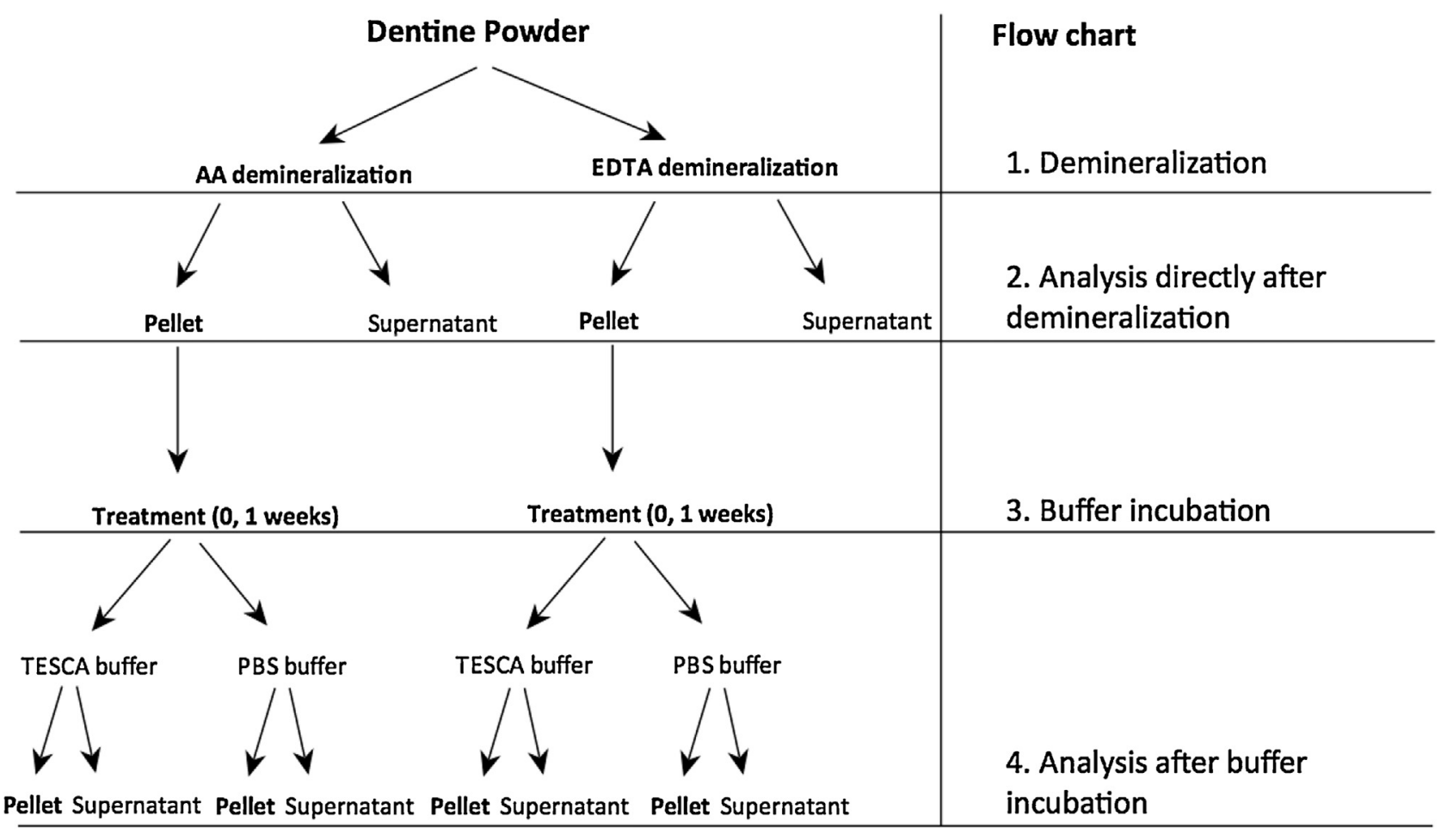

Fig. 1. Schematic overview of the laboratory workflow. 


\subsection{Dentine powder procedure}

The resulting crown dentine was deep-frozen using liquid nitrogen and ground to a fine powder (grain size down to $\sim 5 \mu \mathrm{m}$ ) in a tissue grinder (Retsch Cryomill, Haan, Germany). The powder was subsequently frozen in $-80^{\circ} \mathrm{C}$ until use.

A flowchart describing the demineralization and buffer incubation process is given in Fig. 1.

\subsection{Demineralization process}

\subsubsection{EDTA}

$10 \mathrm{~g}$ of thawed dentine powder was aliquoted into three equal portions and placed in a dialysis tube (Pur-A-Lyzer $10 \mathrm{ml} 12-$ $14 \mathrm{kDa}$; Sigma-Aldrich, St. Louis, MO, USA) with $8 \mathrm{ml}$ of $0.5 \mathrm{M}$ EDTA ( $\mathrm{pH}$ 7.4). The tubes were shaken thoroughly to suspend the powder in the liquid, and placed in a large beaker containing $350 \mathrm{ml}$ of 0.5 M EDTA under constant mixing with a magnetic stirrer (300 rpm). To prevent excessive sedimentation of the powder the tubes were removed from the beaker and agitated twice per $\mathrm{d}$. The EDTA in the beaker was replaced with fresh EDTA every $48 \mathrm{~h}$ and the procedure were repeated until the powder had dissolved 2 entirely. This process demanded four times $48 \mathrm{~h}$ of EDTA treatment (total EDTA treatment $192 \mathrm{~h}$ ). To wash away the EDTA within the dialysis tubes, the liquid in the beaker was changed to laboratory grade water $(350 \mathrm{ml})$ for $24 \mathrm{~h}$ before the water was changed, and was agitated as described above. The water wash procedure was repeated four times (total water wash $192 \mathrm{~h}$ ). The resulting demineralized dentine matrix (DDM; suspended in water) was subsequently frozen in $-80^{\circ} \mathrm{C}$ until use.

\subsubsection{Acetic acid}

$6 \mathrm{~g}$ of thawed dentine powder was aliquoted into three equal portions. Each portion was placed in a dialysis tube as above and $8 \mathrm{ml} 1 \mathrm{M}(\mathrm{pH} \mathrm{2.4)}$ acetic acid (AA) was added. The rest of the demineralization process was performed as described above with EDTA except that EDTA was substituted with AA.

\subsection{DDM retrieval process}

\subsubsection{EDTA derived pellet}

After the demineralization procedure the DDM was suspended in remaining analysis water (from the EDTA washout process). Separation of insoluble DDM was achieved by centrifugation at $5000 \mathrm{rpm}(3913 \mathrm{G})$ for $10 \mathrm{~min}$ in a cooled $\left(4^{\circ} \mathrm{C}\right)$ centrifuge (Hettich Universal 320R; Hettich 1431 rotor, Tuttlingen, Germany). The resulting supernatant was decanted using a pipette. To retrieve as much DDM as possible, the supernatant was recentrifuged, and the resulting pellet added to the pellet from the first centrifugation. The resulting pellets from the three dialysis tubes and from the recentrifuged supernatant were pooled before treatment. Total final pellet wet weight was $4.30 \mathrm{~g}$ (from $10 \mathrm{~g}$ dry dentine powder). The resulting supernatants were also pooled.

\subsubsection{Acetic acid derived pellet}

The same protocol as for the EDTA derived pellet above was used. The resulting pellets from the three dialysis tubes and from the recentrifuged supernatant were pooled before treatment, as were the supernatants. Total final pellet wet weight was $2.8 \mathrm{~g}$ (from $6 \mathrm{~g}$ dry dentine powder).

\subsection{Analyses of DDM directly after demineralization}

\subsubsection{Soluble protein}

Soluble protein concentration in the DDM directly after demineralization (pellet and supernatant) was estimated in order to determine suitable dilutions for SDS-PAGE analysis, using BioRad Protein Assay with the standard protocol for microtiter plates (Bio-Rad, Richmond, CA, USA). The samples were undiluted, except for pellet samples, which were diluted in PBS to $100 \mathrm{mg} / \mathrm{ml}$ and shaken in a vortex mixer. $10 \mu \mathrm{l}$ of blank, standard and sample respectively were put in duplicates in the wells of a microtiter plate, and $200 \mu \mathrm{l}$ Dye Reagent 1:5 was added to each well. The plate was gently shaken (PMS-1000, Grant-Bio, Cambridge, UK) for $10 \mathrm{~min}$ to mix the contents in the wells. Reading of the plate was performed at $595 \mathrm{~nm}$ using a plate reader (ELX-800, Bio-Tek, VT, USA).

\subsubsection{SDS-PAGE}

Uncentrifuged DDM samples ( $10 \mu \mathrm{l} / \mathrm{sample})$ were diluted in $10 \mu \mathrm{l}$ Laemmli SDS sample buffer together with 5\% 2-mercaptoethanol, and heated to $100{ }^{\circ} \mathrm{C}$ for $5 \mathrm{~min}$. From this mixture, $5 \mu$ l were transferred to SDS-PAGE gel in duplicate. Electrophoresis separation of the demineralized dentin proteins was carried out in a $7.5 \%$ SDS-PAGE Criterion TRIS- $\mathrm{HCl}$ precast gel (Bio-Rad Laboratories, Hercules, CA, USA) for $50 \mathrm{~min}$ at $200 \mathrm{~V}$. As collagen control rat tail collagen was used (Sigma-C8897, Sigma-Aldrich). The gels were stained with $0.1 \%$ Coomassie blue on an Orbital shaker (Bellco Glass Inc., New Jersey, USA) for $30 \mathrm{~min}$ and fixed in a $10 \%$ acetic acid and $40 \%$ methanol solution for $30 \mathrm{~min}$, after which they were destained in a $10 \%$ acetic acid and $20 \%$ methanol solution for $3 \times 5 \mathrm{~min}$. The resulting gels were then dried for $24 \mathrm{~h}$ before scanning (Hewlett Packard Scanjet 4c/T, Palo Alto, CA, USA).

\subsection{Treatment of DDM}

\subsubsection{Buffers}

$1000 \mathrm{mg}$ (wet weight) of EDTA- and AA-derived DDM was suspended in $20 \mathrm{ml}$ TESCA buffer ( $50 \mathrm{mM}$ TES, $0.36 \mathrm{mM} \mathrm{CaCl}_{2}, \mathrm{pH}$ 7.4) or PBS buffer $(0.15 \mathrm{M}, \mathrm{pH} 7.2)$ with $\left.0.02 \% \mathrm{NaN}_{3}\right)$ and mixed for $30 \mathrm{~s}$ (Whirlimixer, Fisons Scientific Equipment Limited, London,

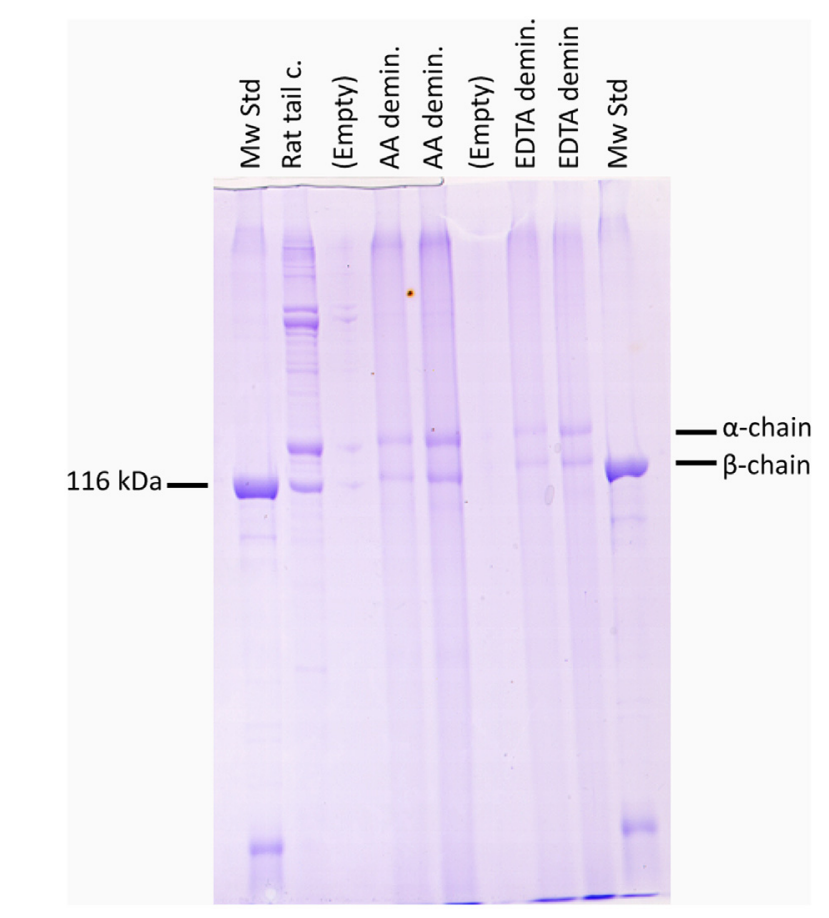

Fig. 2. SDS-PAGE of uncentrifuged DDM

SDS PAGE on 7.5\% Criterion TRIS-HCL gel, $200 \mathrm{~V}, 50 \mathrm{~min} 10 \mu \mathrm{l}$ samples $1: 1$ in sample buffer: Mw Std: Molecular weight standard; Rat tail c.: Rat tail collagen; Acid demin.: Acetic acid demineralized dentine; EDTA demin.: EDTA demineralized dentine. Uncentrifuged samples directly after demineralization was used. 
UK). The resulting suspension was transferred in $2 \mathrm{ml}$ aliquots to a total of nine Eppendorff tubes (in duplicate) and immediately frozen (T0) or incubated in a tube rotator at room temperature for $1 \mathrm{wk}$ (T1) before freezing in $-80^{\circ} \mathrm{C}$ until further analysis.

\subsection{Post-treatment analyses}

\subsubsection{Preparation of buffer incubated samples}

The DDM frozen in TESCA and PBS buffer (T0 and T1) was thawed in room temperature. Separation was achieved by centrifugation at $5000 \mathrm{rpm}(2711 \mathrm{G})$ for $10 \mathrm{~min}$ in a cooled centrifuge (Hettich). The resulting supernatant was decanted using a pipette and saved for further analysis. Each pellet was then resuspended in $1000 \mu \mathrm{l}$ PBS buffer. The suspended pellets were used for all subsequent analyses.

\subsubsection{SDS-PAGE}

The thawed DDM from the post-treatment samples were analyzed using the same SDS-PAGE method as described above.

\subsubsection{Collagen}

The collagen concentration was estimated by measuring the concentration of HYP using the QuickZyme Total Collagen Assay (QuickZyme Biosciences, Leiden, The Netherlands) according to the manufacturer's instructions. This was done for T0 pellet and T0 and T1 supernatant samples. Briefly, standards and samples were hydrolyzed in a thermoblock (Stuart Block Heater SBH130D/3, Bibby Scientific Limited, Staffordshire, UK) in $6 \mathrm{M} \mathrm{HCl}$ at $95^{\circ} \mathrm{C}$ for $20 \mathrm{~h}$. Hydrolyzed standards and samples were transferred to a 96well assay plate and assay buffer was added. The plate was incubated at room temperature for $20 \mathrm{~min}$ on a plate shaker. Detection reagent was added and the plate incubated for $60 \mathrm{~min}$ in $60^{\circ} \mathrm{C}$, cooled on ice to room temperature, and read at $540 \mathrm{~nm}$ in a plate reader (ELX-800).

\subsection{4. $M M P-8$}

Levels of active MMP-8 levels in the pellet samples were analyzed using the QuickZyme Human MMP-8 activity assay (QuickZyme Biosciences) according to the manufacturer's instructions. Briefly, anti-MMP-8 was pipetted to a pre-coated (F(ab')2 goat anti-mouse) 96 -well assay plate and incubated in $37^{\circ} \mathrm{C}$ for $2 \mathrm{~h}$. After washing, standards and samples were added to the plate and the plate was incubated at $2-8{ }^{\circ} \mathrm{C}$ over night. After new washing, assay buffer was added and the plate was incubated at $37^{\circ} \mathrm{C}$ for $1 \mathrm{~h}$ before adding detection reagent. After $6 \mathrm{~h}$ the plate was read at $405 \mathrm{~nm}$ in a plate reader (ELX-800).

\subsection{Statistical analysis}

All statistical analyses were performed using two-way ANOVA analysis using IBM SPSS 20.0 software for Mac (IBM Corporation, Somers, New York, USA). A P value of less than 0.05 was regarded as statistically significant.

\section{Results}

\subsection{Analysis of DDM directly after demineralization}

\subsubsection{Soluble protein assay of original DDM}

Directly after demineralization, soluble protein levels of the DDM and supernatant were determined. The protein levels were slightly more pronounced in the EDTA derived DDM when the full sample was analyzed directly after demineralization. When fractioned into pellet and supernatant, there was a marked difference in the distribution pattern of proteins.

\subsubsection{SDS-PAGE of original DDM}

The SDS-PAGE gel from directly after demineralization exhibited distinct collagen type 1 bands in all samples. The characteristic $130 \mathrm{kDa}$ and $115 \mathrm{kDa}$ collagen type 1 bands corresponding to the $\alpha 1$ - and $\alpha 2$-chains from human type 1 collagen, respectively, were present in all instances (Fig. 2). This demonstrated the presence of collagen type 1 in all fractions of the original DDM, although in different concentrations.

\subsection{Analysis of DDM after buffer treatment}

\subsubsection{SDS-PAGE at start (TO) and after buffer incubation (T1)}

The SDS-PAGE gel from after the buffer treatment displayed the same distinct collagen type 1 bands as the uncentrifuged DDM in all instances, regardless of buffer used (PBS or TESCA) or treatment time (no incubation or $1 \mathrm{wk}$ incubation), indicating that collagen type $1 \alpha 1$ - and $\alpha 2$ - chains were present in all samples (data not shown).

\subsubsection{Collagen measurements after incubation in buffer (TO)}

HYP levels in pellet for the different demineralization methods, buffer solutions and fractions are given in Table 1. AA demineralization yielded approximately twice the concentration of HYP compared to EDTA demineralization. The difference due to demineralization method was highly statistically significant $(\mathrm{p}<0.001$, Table 1$)$.

\subsubsection{Influence of buffers on collagen levels after incubation (T0 to T1)}

The total HYP concentration increased in the supernatant after incubation in room temperature for $1 \mathrm{wk}$ (Fig. 3a and b). This increase was statistically significant $(p<0.001)$, as was buffer treatment $(\mathrm{p}<0.001)$ and the combination of demineralization method/buffer treatment $(\mathrm{p}=0.046)$.

\subsubsection{MMP-8 assay after buffer treatment}

Total MMP-8 levels in pellet samples for the different demineralization methods, buffer solutions and treatment times are given in Table 2. No data is given for MMP-8 in the buffer supernatants, as levels were below the detection limit. MMP8 levels were higher at the start of the buffer treatment period (T0) than after $1 \mathrm{wk}(\mathrm{T} 1)$.

AA-derived DDM displayed similar MMP-8 levels regardless of buffer. EDTA-derived DDM on the other hand displayed much elevated MMP-8 levels in TESCA buffer at T0, but this had decreased to a level similar to PBS at T1 (Fig. 4a and b).

The active MMP-8 concentration change over time was statistically significant $(\mathrm{p}<0.01$ ), as was demineralization method $(p=0.02)$ and the combination of demineralization/treatment $(p=0.046)$ in a statistical model encompassing the variables time, demineralization method and buffer, with MMP-8 as the dependent variable.

Table 1

Hydroxyproline (HYP) in pellet at T0.

\begin{tabular}{llll}
\hline Treatment buffer & Demineralization method & $\mu \mathrm{\mu g} / \mathrm{ml}$ & \\
\cline { 3 - 4 } & & Mean & \pm SD \\
\hline \multirow{2}{*}{ TESCA } & EDTA & 1017.33 & 78.79 \\
& AA & 2396.74 & 331.15 \\
PBS & EDTA & & \\
& AA & 1692.50 & 126.22 \\
& & 2784.07 & 488.64 \\
\hline
\end{tabular}

Mean concentration of HYP in samples directly after adding incubation buffer. $\mathrm{N}=3$ per treatment (in duplicate). 
(a) TESCA buffer

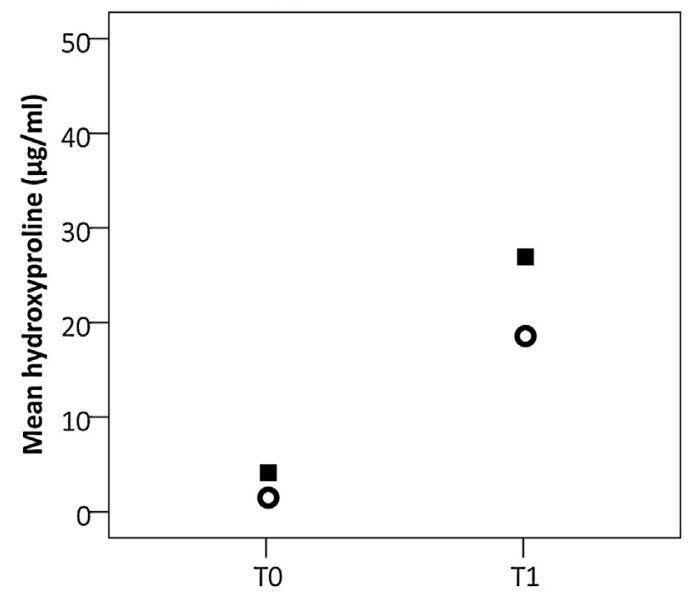

(b) PBS buffer

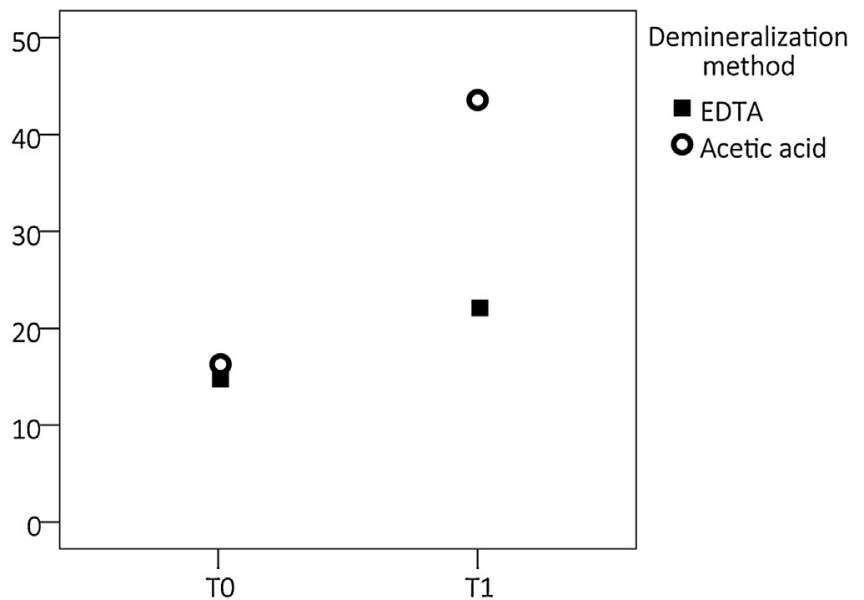

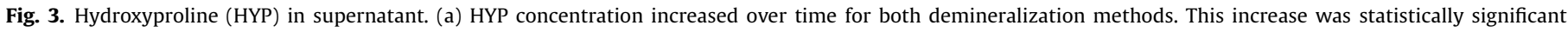

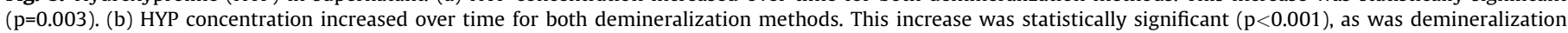
method $(\mathrm{p}<0.001)$.

\section{Discussion}

This study describes a new method for dentine powder demineralization without loss of enzyme bioactivity. The use of a dialysis method also allows for retention of the majority of the DDM components that otherwise would be partly lost due to the demineralization method. Dentine powder is rarely used due to difficulties in controlling the collection of samples. However, our finding that dentine powder derived DDM collagen degrades over time is confirmed by the results of a previous study using EDTAand phosphoric acid demineralized human dentine beams (Carrilho et al., 2009), which would indicate that dentine powder is well suited for this type of research.

Enzymatic activity was preserved to a significant extent as demonstrated by MMP-8 activity and solubilization of insoluble collagen. The system allowed for collection of soluble and insoluble material simultaneously, and harvesting of the insoluble gelatinatious material included scraping of the inside of the dialysis vessel in order to retrieve all possible DDM.

The utilization of a dialysis system during demineralization allowed for the retention of the major part of the constituents of the DDM, mainly collagen type 1 but also proteoglycans and noncollagenous proteins (Tjäderhane et al., 2013a). The pore size of $12 \mathrm{kDa}$ allowed for retention of molecules like the $\alpha 1$ - and $\alpha 2$ chains of collagen type 1 . MMP-8 split the collagen molecules in two specific fragments (1/4 and $3 / 4$ fragments of the intact molecule) that cannot pass the membrane (Tjäderhane et al., 2013b). MMP-8 and proMMP-8 (molecular weights of 65 and $85 \mathrm{kDa}$, respectively) were also retained. If collagen degradation occurred during the demineralization phase, the degradation products such as collagen telopeptides and hydroxyproline could traverse the membrane, likely leading to some loss of smaller molecular weight DDM components such as osteocalcin (6 kDa). However, the purpose of this study was to collect and describe insoluble DDM, which contained collagen including bound proteins, for future intervention assays. When subsequent analyses were made of the crude uncentrifuged DDM, collagen was demonstrated in all samples, using SDS-PAGE electrophoresis (Fig. 2).

The protein analyses were performed to be able to compute sample size for the SDS-PAGE analysis and should be interpreted with caution since the method used only measure the soluble protein fraction correctly. Even so, it is interesting to note that the distribution of the soluble proteins in EDTA- and AA-demineralized samples were markedly different. With EDTA, about $80 \%$ was detected from the supernatant, while AA-demineralized samples presented only $20 \%$. This finding is interesting; many of the demineralizing methods described in the literature are aimed at purifying dentine proteins for identification, and while they might be excellent for demineralization, their respective effects on the retention of noncollagenous proteins and the bioactivity of enzymes are little known.

Because EDTA is a chelator that can be used at neutral $\mathrm{pH}$, it is often considered an optimal demineralization agent of teeth for immunohistochemistry (Sanjai et al., 2012). Furthermore, EDTA is a potent MMP inhibitor, preventing activation of MMP during the demineralization stage (Toledano, Yamauti, Osorio \& Osorio, 2012). It has also been demonstrated to possess antimicrobial properties (Banin, Brady, \& Greenberg, 2006).

Comparative studies between different decalcifying methods in terms of preservation or loss of dentinal proteins are lacking. Earlier dentine demineralization studies have often utilized $\mathrm{HCl}$ in combination with guanidine, which is a potent protein-denaturing agent (Linde, Bhown, \& Butler, 1980). AA was chosen for this study based on its weaker and presumably less denaturing properties, as well as its frequent appearance in carious lesions (Hojo, Komatsu, Okuda, Takahashi, \& Yamada, 1994). Theoretically, AA probably activates MMPs due to its low $\mathrm{pH}$ (2.4), but since MMPs have a $\mathrm{pH}$ optimum of 7, no or very low activity would take place during the demineralization stage (Okada et al., 1995). The activity could, however, increase during dialysis with water in the washout stage of the dialysis.

The total collagen assessments were performed using HYP as an indicator of collagen levels after complete hydrolysis of the

Table 2

Active MMP-8 in pellet at T0.

\begin{tabular}{llll}
\hline Treatment buffer & Demineralization method & \multicolumn{2}{l}{$\mathrm{ng} / \mathrm{ml}$} \\
\cline { 3 - 4 } & & Mean & $\pm \mathrm{SD}$ \\
\hline \multirow{2}{*}{ TESCA } & EDTA & 5.92 & 1.07 \\
& AA & 4.86 & 1.64 \\
\multirow{2}{*}{ PBS } & EDTA & 1.81 & 1.68 \\
& AA & 5.10 & 1.77 \\
\hline
\end{tabular}

Mean concentration of active MMP-8 in samples directly after adding incubation buffer. $\mathrm{N}=3$ per treatment (in duplicate). 
(a) TESCA buffer

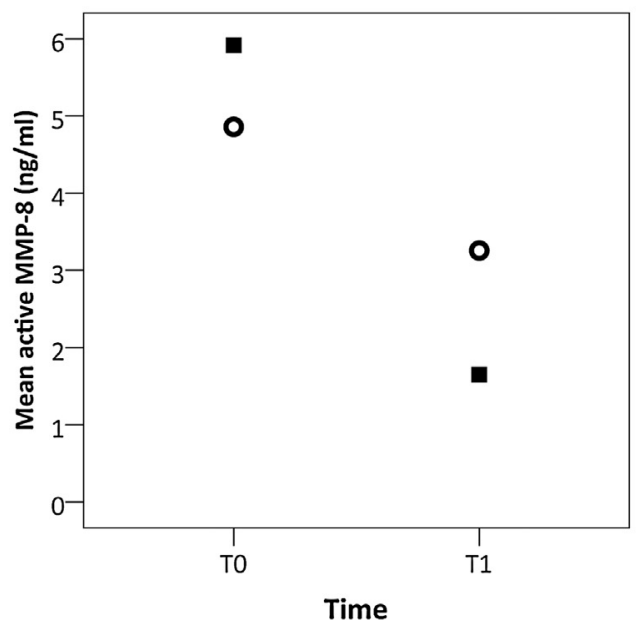

(b) PBS buffer

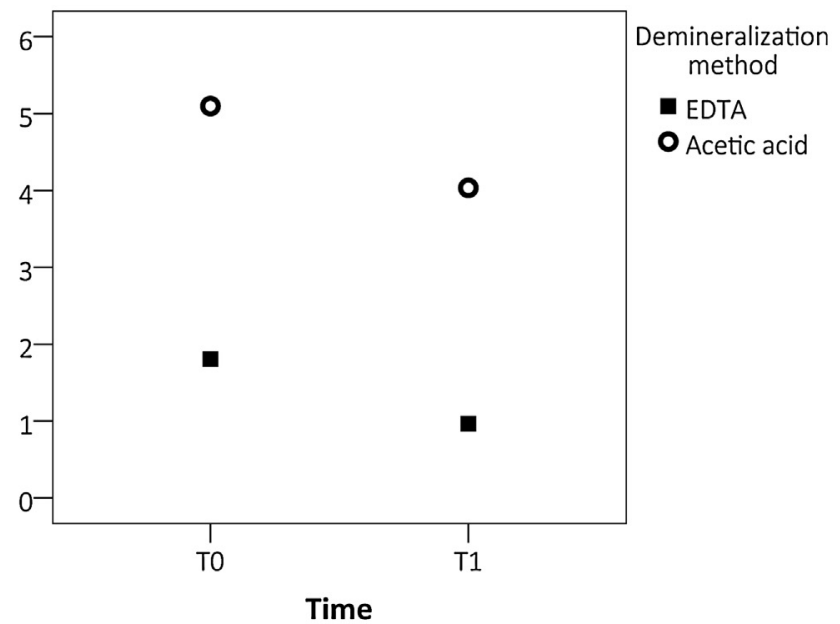

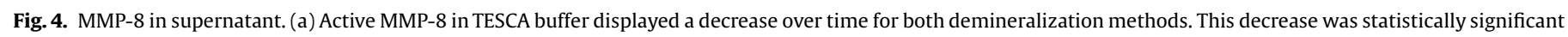

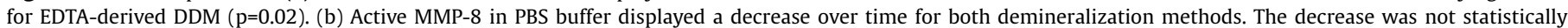
significant.

samples. As can be seen in Table 1, the AA-derived DDM pellet levels of total collagen were twice as high as the corresponding EDTA-derived DDM. An explanation for this could be that the resulting DDM from the different demineralization methods has different water retention capacity, which would influence the wet pellet weight. We did not perform complete water removal by lyophilization, as the process might harm bioactive molecules, and especially enzymes (Roy \& Gupta, 2004). In addition, repeated freezing and thawing could activate MMPs (Nishitani et al., 2006).

The HYP levels increased in the supernatants of all samples (Fig. 3a and b), which indicates that collagen is solubilized over time. For the AA-derived DDM samples a difference in PBS- buffer was demonstrated at T1 (Fig. 3a and b). The reason for this could be that the AA demineralization sequence has started hydrolysis of the molecules, making them more prone to further degradation. However, the most important finding regarding HYP is that solubilization of DDM collagen can be demonstrated in dentine powder samples, similar to dentine beams (Carrilho et al., 2009).

Table 2 presents the endogenously activated MMP-8 data from the buffer-treated pellets. Corresponding supernatant data is not presented as the levels were below detection level. For AA-derived DDM the buffer did not affect the MMP-8 values, but in the EDTAderived DDM group the MMP-8 values from PBS treated samples were much lower, indicating that EDTA demineralization could effectively inhibit MMP activity and that $\mathrm{Ca}^{2+}$ from the TESCA buffer was needed to reactivate MMP-8. In contrast, AA demineralization does not seem to inhibit MMP-8 as much. This is supported by a previous study where dentine powder was demineralized using four different agents, and AA yielded about the double amount of active MMPs (MMP-2 and -9) after demineralization (Mazzoni et al., 2007). One explanation for higher MMP-8 activity in AA-derived DDM could be acid activation (Tjäderhane et al., 1998), or trace amounts of $\mathrm{Ca}^{2+}$ being present. This was mirrored by the fact that in EDTA-derived DDM the use of TESCA buffer enhanced MMP-8 activity to a great extent.

Fig. 4 reveals that MMP-8 activity decreases with time, in particular for EDTA-derived DDM in TESCA buffer. This decrease in MMP activity was statistically significant (Fig. 4a) and could be attributed to depletion of necessary reagents or enzyme activity regulation by feedback inhibition mediated by degradation products. These enzyme activity-limiting processes could differ between the buffers used.
The presence of active MMP- 8 and simultaneous degradation of collagen in this experiment suggests that MMP- 8 can contribute to collagen breakdown. However, the action of other endogenic enzymes was not assessed. Cysteine cathepsins have been implicated in both activation of MMPs and collagen degradation (Nascimento et al., 2011), but inclusion of these enzymes was beyond the scope of this study.

\section{Conclusions}

The presented demineralization methods both provided insoluble DDM substrates suitable for further intervention studies. However, it was found that the substrates differed depending on the demineralization method and buffers used. It is conceivable that yet other factors like $\mathrm{pH}$ challenge, temperature and time might also affect the final properties of the substrate and that the most appropriate demineralization method might depend on what compound is sought to be analyzed. This needs to be studied further in order to find an optimal technique to generate DDM with retained proteins as well as enzymatic bioactivity.

\section{Conflicts of interest}

The authors declare no conflicts of interest.

\section{Acknowledgements}

The authors would like to acknowledge Dr. Per-Erik Isberg, Department of Statistics, Lund University, for excellent statistical advice. Funded by Faculty Grants and the Swedish Dental Society. The funders had no role in study design, data collection and analysis, decision to publish, or preparation of the manuscript. Conceived and designed the study: AHL, DE, LT. Performed the dentine collection: VP. Performed the experiments: AHL, KH. Analyzed the data: AHL. Wrote the paper: AHL (main author), DE, LT, VP.

\section{References}

Banin, E., Brady, K. M., \& Greenberg, E. P. (2006). Chelator-induced dispersal and killing of pseudomonas aeruginosa cells in a biofilm. Applied and Environmental Microbiology, 72(3), 2064-2069. 
Carrilho, M. R., Tay, F. R., Donnelly, A. M., Agee, K. A., Tjäderhane, L., Mazzoni, A., et al (2009). Host-derived loss of dentin matrix stiffness associated with solubilization of collagen. Journal of Biomedical Materials Research Part B, Applied Biomaterials, 90(1), 373-380.

Chaussain-Miller, C., Fioretti, F., Goldberg, M., \& Menashi, S. (2006). The role of matrix metalloproteinases (mmps) in human caries. Journal of Dental Research, 85(1), 22-32.

Hedenbjörk-Lager, A., Bjørndal, L., Gustafsson, A., Sorsa, T., Tjäderhane, L., Akerman, S., et al. (2015). Caries correlates strongly to salivary levels of MMP-8. Caries Research, 49(1), 1-8.

Hojo, S., Komatsu, M., Okuda, R., Takahashi, N., \& Yamada, T. (1994). Acid profiles and $\mathrm{pH}$ carious dentin in active and arrested lesions. Journal of Dental Research, 73 (12), 1853-1857.

Katz, S., Park, K. K., \& Palenik, C. J. (1987). In-vitro root surface caries studies. Journal of Oral Medicine, 42(1), 40-48.

Linde, A., Bhown, M., \& Butler, W. T. (1980). Noncollagenous proteins of dentin. A reexamination of proteins from rat incisor dentin utilizing techniques to avoid artifacts. The Journal of Biological Chemistry, 255(12), 5931-5942.

Mazzoni, A., Mannello, F., Tay, F. R., Tonti, G. A., Papa, S., Mazzotti, G., et al. (2007). Zymographic analysis and characterization of MMP-2 and -9 forms in human sound dentin. Journal of Dental Research, 86(5), 436-440.

Nascimento, F. D., Minciotti, C. L., Geraldeli, S., Carrilho, M. R., Pashley, D. H., Tay, F. R., et al. (2011). Cysteine cathepsins in human carious dentin. Journal of Dental Research, 90(4), 506-511.

Nishitani, Y., Yoshiyama, M., Wadgaonkar, B., Breschi, L., Mannello, F., Mazzoni, A., et al. (2006). Activation of gelatinolytic/collagenolytic activity in dentin by selfetching adhesives. European Journal of Oral Sciences, 114(2), 160-166.

Nordbø, H., Leirskar, J., Ngo, H., Mount, G. J., \& Wahlgren, J. (2003). The influence of a matrix metalloproteinase on the remineralization of artificially demineralized dentin. Oral Health \& Preventive Dentistry, 1(4), 267-272.

Okada, Y., Naka, K., Kawamura, K., Matsumoto, T., Nakanishi, I., Fujimoto, N., et al. (1995). Localization of matrix metalloproteinase 9 (92-kilodalton gelatinase/ type IV collagenase = gelatinase B) in osteoclasts: implications for bone resorption. Laboratory Investigation; A Journal of Technical Methods and Pathology, 72(3), 311-322.

Roy, I., \& Gupta, M. N. (2004). Freeze-drying of proteins: some emerging concerns. Biotechnology and Applied Biochemistry, 39(Pt 2), 165-177.

Sanjai, K., Kumarswamy, J., Patil, A., Papaiah, L., Jayaram, S., \& Krishnan, L. (2012). Evaluation and comparison of decalcification agents on the human teeth. Journal of Oral and Maxillofacial Pathology: JOMFP, 16(2), 222-227.
Sorsa, T., Tjäderhane, L., \& Salo, T. (2004). Matrix metalloproteinases (mmps) in oral diseases. Oral Diseases, 10(6), 311-318.

Sulkala, M., Tervahartiala, T., Sorsa, T., Larmas, M., Salo, T., \& Tjäderhane, L. (2007). Matrix metalloproteinase-8 (MMP-8) is the major collagenase in human dentin. Archives of Oral Biology, 52(2), 121-127.

Sulkala, M., Wahlgren, J., Larmas, M., Sorsa, T., Teronen, O., Salo, T., et al. (2001). The effects of MMP inhibitors on human salivary MMP activity and caries progression in rats. Journal of Dental Research, 80(6), 1545-1549.

Takahashi, N., \& Nyvad, B. (2011). The role of bacteria in the caries process: ecological perspectives. Journal of Dental Research, 90(3), 294-303.

Tezvergil-Mutluay, A., Mutluay, M., Seseogullari-Dirihan, R., Agee, K. A., Key, W. O. Scheffel, D. L., et al. (2013). Effect of phosphoric acid on the degradation of human dentin matrix. Journal of Dental Research, 92(1), 87-91.

Tjäderhane, L., Larjava, H., Sorsa, T., Uitto, V. J., Larmas, M., \& Salo, T. (1998). The activation and function of host matrix metalloproteinases in dentin matrix breakdown in caries lesions. Journal of Dental Research, 77(8), 1622-1629.

Tjäderhane, L., Nascimento, F. D., Breschi, L., Mazzoni, A., Tersariol, I. L., Geraldeli, S. et al. (2013). Optimizing dentin bond durability: control of collagen degradation by matrix metalloproteinases and cysteine cathepsins. Dental Materials: Official Publication of the Academy of Dental Materials, 29(1), 116-135.

Tjäderhane, L., Nascimento, F. D., Breschi, L., Mazzoni, A., Tersariol, I. L., Geraldeli, S. et al. (2013). Strategies to prevent hydrolytic degradation of the hybrid layer-a review. Dental Materials: Official Publication of the Academy of Dental Materials, 29(10), 999-1011.

Tjäderhane, L. Sulkala, M., Sorsa, T, Teronen, O, Larmas, M. \& Salo, T. (1999). The effect of MMP inhibitor metastat on fissure caries progression in rats. Annals of the New York Academy of Sciences, 878, 686-688.

Toledano, M., Yamauti, M., Osorio, E., \& Osorio, R. (2012). Zinc-inhibited mmpmediated collagen degradation after different dentine demineralization procedures. Caries Research, 46(3), 201-207.

Visse, R., \& Nagase, H. (2003). Matrix metalloproteinases and tissue inhibitors of metalloproteinases: structure, function, and biochemistry. Circulation Research, 92(8), 827-839.

van Strijp, A. J., van Steenbergen, T. J., de Graaff, J., \& ten Cate, J. M. (1994). Bacterial colonization and degradation of demineralized dentin matrix in situ. Caries Research, 28(1), 21-27.

van Strijp, A. J., van Steenbergen, T. J., \& ten Cate, J. M. (1997). Bacterial colonization of mineralized and completely demineralized dentine in situ. Caries Research, 31 (5), 349-355. 